COMMENT. The results of surgical treatment of the above 26 patients are reported in the same journal (Palmini A et al. Ann Neurol Dec $1991 ; \underline{30}: 750-757)$. Good or excellent seizure control was achieved in $42 \%$ and moderate control in $25 \%$. The extent of lesion removed was most strongly correlated with the surgical outcome; $77 \%$ receiving complete or $50 \%$ excision of the lesion had a good prognosis. When excision of epileptogenic tissue was based on scalp EEG and electrocorticography studies there was no correlation with surgical outcome. The lack of specificity of the MRI in the diagnosis of tuberous sclerosis in this study is also alluded to by Fryer AE, Geneticist at the Royal Liverpool Hospital, England in an editorial concerning tuberous sclerosis (J Roy Soc Med Dec 1991; $\underline{84: 699-701) .}$ Although MRI is more sensitive than CT, CT is more specific and potentially less confusing in interpretation. A thorough clinical examination including ophthalmoscopy and ultraviolet light examination of the skin is essential to exclude the diagnosis in anyone suspect or at risk. Prenatal diagnosis is only possible by fetal echocardiography but this examination is unreliable before 26 weeks and has a high rate of false negatives.

\title{
MMR IMMUNIZATION AND FEBRILE SEIZURES
}

The risk of seizures following MMR or MR immunization is evaluated in a retrospective cohort study conducted among 18,364 Tennessee children from The Department of Preventive Medicine, Vanderbilt University School of Medicine, Nashville, TN. Of 100 children $(0.5 \%)$ with a confirmed seizure between the first MMR or MR and age 36 months, 77 had febrile seizures, 15 afebrile seizures and 8 acute symptomatic seizures. The incidence of febrile seizures was $4.3,5.1$, and 2.8 per 1,000 person-years in the 3 age groups, 361-540, 541-720, and 721-1,080 days, respectively. 4 children had febrile seizures in the 7-14 days following MMR or MR immunization, compared with 72 in the interval 30 or more days following MMR or MR with a relative risk of 2.1. The authors concluded that the increase in febrile seizures in the 7-14 day interval following MMR was coincident with the occurrence of fever postimmunization (Griffin MR et al. Risk of seizures after measles-mumps-rubella

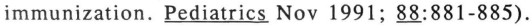

COMMENT. This review of seizure occurrence was confined to those patients seen at a hospital and would not include seizures treated at home or at outpatient facilities. The authors admit that the ascertainment of seizures was incomplete but would include more serious seizures or neurologic events.

Measles was the cause of fever in $1.2 \%$ of 7,000 cases of febrile seizures reported in the world literature between 1929 and 1964 (Millichap JG, Febrile convulsions 1968 Macmillan, New York). 